\title{
Quantitative Radiography of Magnetic Fields Using Neutron Spin Phase Imaging
}

\author{
F. M. Piegsa, ${ }^{1,2, *}$ B. van den Brandt, ${ }^{1}$ P. Hautle, ${ }^{1}$ J. Kohlbrecher, ${ }^{1}$ and J. A. Konter ${ }^{1}$ \\ ${ }^{1}$ Paul Scherrer Institute, $\mathrm{CH}-5232$ Villigen PSI, Switzerland \\ ${ }^{2}$ Physics Department, Technische Universität München, D-85748 Garching, Germany
}

(Received 15 December 2008; published 9 April 2009)

\begin{abstract}
We report on a novel neutron radiography technique that uses the Ramsey principle, a method similar to neutron spin echo. For the first time quantitative imaging measurements of magnetic objects and fields could be performed. The strength of the spin-dependent magnetic interaction is detected by a change in the Larmor precession frequency of the neutron spins. Hence, one obtains in addition to the normal attenuation radiography image a so-called neutron spin phase image, which provides a two-dimensional projection of the magnetic field integrated over the neutron flight path.
\end{abstract}

Introduction.-Radiography and tomography with neutrons have become well-established techniques to image macroscopic objects of interest in various research disciplines [1]. Neutrons interact with the nuclei in matter via the strong interaction and therefore deliver complementary information to $\mathrm{x}$ rays, which are sensitive to the electron density distribution. Spatial resolutions of down to a few tens of micrometers can be reached using state-of-the-art techniques [2-5]. So far in the history of these techniques the property of the spin of the neutron and the associated magnetic momentum $\mu_{n}$ has been neglected.

As neutrons do not possess an electric charge, but a magnetic moment, they are the ideal probes to investigate magnetic interactions. This is the basis of solid state research of magnetic samples with polarized neutrons, e.g., examining transitions between different magnetic phases or magnetic excitations. These scattering techniques are complementary to neutron radiography as they are probing the momentum space and the energy transfer instead of the real space. The radiography technique presented here combines a method well known in neutron scattering $[6,7]$ with a typical imaging setup.

Ramsey's technique of separated oscillating fields is a method sensitive to spin-dependent interactions of particles with external fields, detected as a spin precession angle $\varphi[8,9]$, and is therefore similar to spin echo techniques, e.g., pulsed nuclear magnetic resonance (NMR) or neutron spin echo (NSE) [10]. Ramsey's method adapted to neutrons can be employed to observe interactions between neutron spins and magnetic or pseudomagnetic fields $[11,12]$, where the latter is a formal description of the spin-dependent interaction between neutrons and polarized nuclei [13]. A neutron Ramsey apparatus consists ideally of a homogeneous steady magnetic field $\vec{B}_{0}$, where the spins of a passing monoenergetic polarized neutron beam get nonadiabatically flipped twice by $90^{\circ}$ by two phase-locked fields oscillating perpendicularly to $\vec{B}_{0}$ with an angular frequency $\omega(\pi / 2$-spin flippers). Between the spin flips the neutron spins precess freely in the plane perpendicular to the steady field with the neutron Larmor frequency $\omega_{0}=-\gamma_{n} B_{0}$, where $\gamma_{n}$ is the gyromagnetic ratio of the neutron. Behind the second $\pi / 2$-spin flipper the neutron beam polarization gets analyzed by a neutron spin filter, which is placed in front of the neutron detector. Successive scanning of the angular frequency $\omega$ close to $\omega_{0}$ results in a sinusoidal intensity oscillation in the detector (Ramsey pattern). Any additional precession angle $\varphi$ of the neutron spins due to a sample shifts the phase of the Ramsey pattern by the same value. The precession angle $\varphi_{m}$ due to magnetic interaction is given by

$$
\varphi_{m}=\frac{m_{n} \gamma_{n} B}{h} \lambda s,
$$

where $\lambda$ and $m_{n}$ are the neutron de Broglie wavelength and mass, $s$ is the length of the flight path in which the neutron spins sense a magnetic field $B$, and $h$ is Planck's constant. Previously, NSE has been combined with a position sensitive detector [14-16]. However, these authors were not particularly interested in spatially resolved images, but rather in bulk quantities of the investigated samples. More important, due to the typically long sample-detector distance of spin echo instruments anyway only a poor resolution would have been attained. Reference [17] tries to overcome this drawback and determines the local magnetization of a single crystal specimen by scanning the sample with a beam of $0.1 \mathrm{~mm}^{2}$ in cross section. Obviously, this method is highly inefficient in terms of measuring time compared to the here-presented neutron spin phase imaging (NSPI) technique, which allows illumination of the entire sample and provides a submillimeter spatial resolution.

Experiments and results.-To demonstrate the technique, measurements were performed on two different ferromagnetic precision steel foils with thicknesses of $(20 \pm 2) \mu \mathrm{m}$ and $(40 \pm 3) \mu \mathrm{m}$, respectively [18]. The foils were oriented along the magnetic field $\vec{B}_{0}$ perpendicular to the penetrating neutron beam, leaving a gap of about $4 \mathrm{~mm}$ between them. Figures 1(a)-1(d) show radiography images of the foils for four different spin flipper frequencies, where the $20 \mu \mathrm{m}(40 \mu \mathrm{m})$ foil is situated on 
a
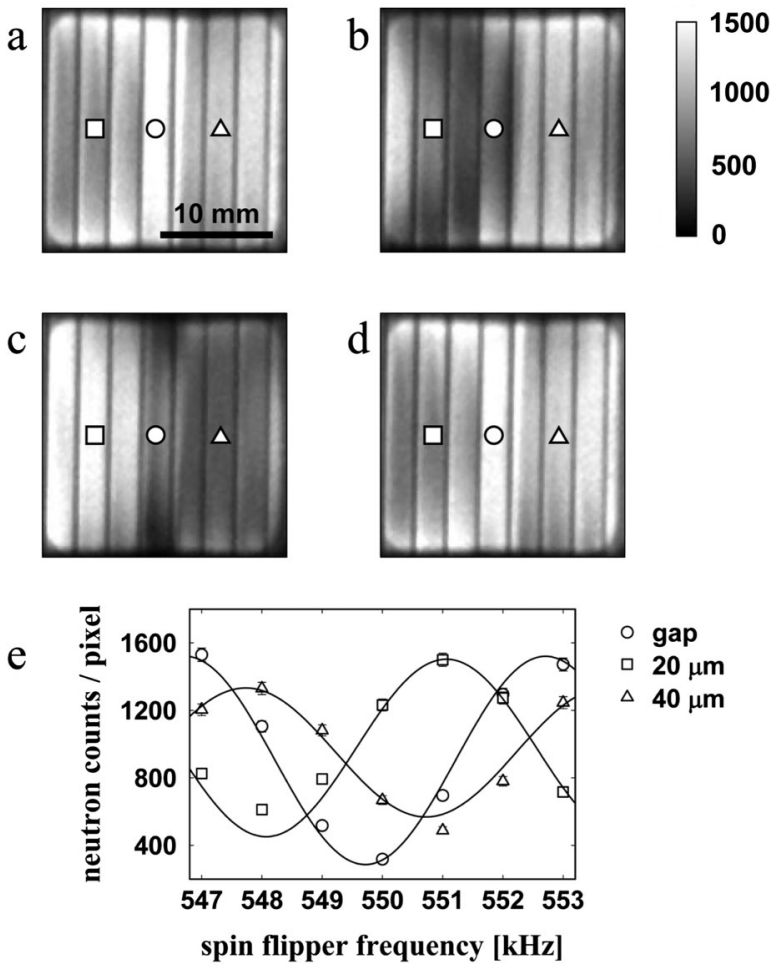

FIG. 1. Radiography images of ferromagnetic steel foils using neutrons of $\lambda_{0}=0.6 \mathrm{~nm}$ at different $\pi / 2$-spin flipper frequencies: (a) $547 \mathrm{kHz}$, (b) $549 \mathrm{kHz}$, (c) $551 \mathrm{kHz}$, and (d) $553 \mathrm{kHz}$. Each image has a size of $165 \times 165$ pixels, which corresponds to about $21.5 \times 21.5 \mathrm{~mm}^{2}$. The vertical stripes with lowered intensity result from the geometry of the transmission spin analyzer. (e) Intensity modulation in three different areas (averaged over $12 \times 12$ pixels) of the images as a function of the spin flipper frequency and sinusoidal fits: gap (circle), $20 \mu \mathrm{m}$ foil (square), and $40 \mu \mathrm{m}$ foil (triangle).

the left (right) side of the images. One can clearly recognize a position dependent modulation of the detected neutron intensity as a function of the spin flipper frequency. To illustrate this effect the intensities of three areas at different positions of the image have been averaged and plotted in Fig. 1(e). Besides the phase shifts of the modulated signals one also observes a damping of the amplitudes, mainly caused by the finite width of the neutron wavelength spectrum [12] and further by possible inhomogeneities of the magnetization of the foils. However, the decrease of the signal offset delivers the standard neutron attenuation image; i.e., the attenuation information of the sample is already included in these measurements. Fitting such signals with a sinusoidal function and plotting the phase values $\varphi(x, z)$ for each pixel yields the so-called NSPI image. The phase shift $\Delta \varphi(x, z)$ due to the sample is obtained by subtracting the NSPI image of the empty beam from the one with the sample. The corresponding NSPI images of the ferromagnetic foils and of the empty beam are both generated from a set of 11 radiography images taken at different spin flipper frequencies from 545 to $555 \mathrm{kHz}$ with an exposure time of about one minute each. This yields a statistical accuracy of the phase retrieval of about $\pm 5^{\circ}$ for each pixel. The resulting phase shift image modulo $360^{\circ}$ together with a horizontal cut through the image are shown in Fig. 2. Note that the phase shift due to a ferromagnetic sample is always positive. To determine the exact phase shift one performs this measurement for different neutron wavelengths $\lambda_{0}$ and plots the phase shift as a function of $\lambda_{0}$, which yields a line through the origin, as presented in Fig. 3(a). From the slope of the linear fit and the thickness of the foils one can determine the local magnetization of the foils averaged along the neutron flight path. Equation (1) leads for this example to a local magnetization $\mu_{0} M$ of $(1.08 \pm 0.08 \pm 0.11) \mathrm{T}$ for the $20 \mu \mathrm{m}$ foil and $(1.81 \pm$ $0.02 \pm 0.14) \mathrm{T}$ for the $40 \mu \mathrm{m}$ foil, where the errors are due to the linear fit and the thickness tolerances of the foils, respectively. These results can be compared with hysteresis curves of the shim steel foils taken with a physical property measurement system (PPMS) [19], which are presented in Fig. 3(b). The magnetizations $\mu_{0} M$ are about 0.82 and $1.55 \mathrm{~T}$ at an external magnetic field of $19 \mathrm{mT}$ (compare technical details). This is close to the local magnetization values obtained with the NSPI technique. Possible reasons for the differences between the two methods are first that the NSPI technique determines the magnetization locally and the PPMS averages over the whole sample. Second, the samples used for the PPMS have to be much smaller in size and have usually a different shape due to geometrical restrictions of the setup, which can cause deviations in the demagnetization factors and the hysteresis curve. Last, in the neutron measurement the steel foils touched the ferromagnetic steel plates of the NSPI setup (like in an iron yoke) to reduce fringe fields of the sample and therefore might have been magnetized close to the saturation magnetizations $\mu_{0} M_{s}$ of about 1.08 and $1.93 \mathrm{~T}$, respectively.
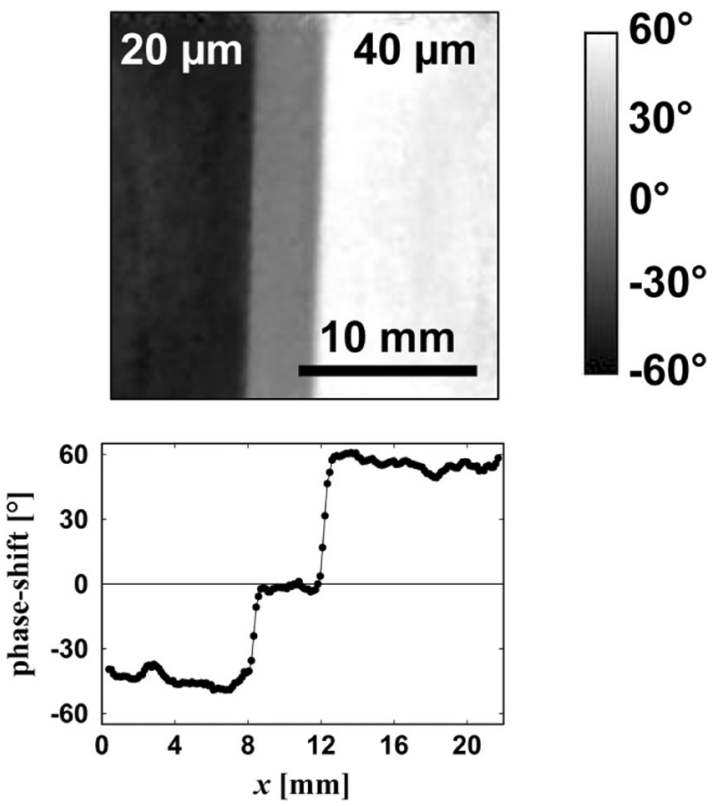

FIG. 2. Resulting NSPI image of the two different ferromagnetic steel foils and plot of a horizontal cut. 


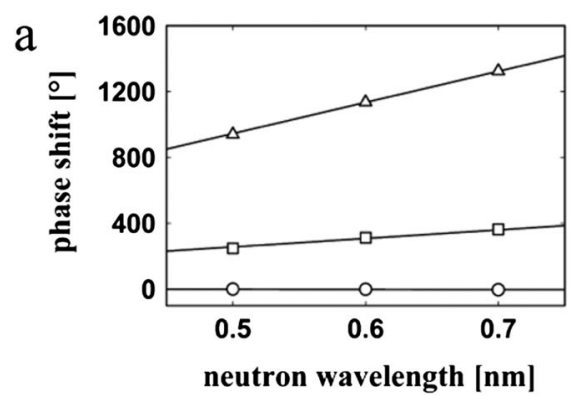

b

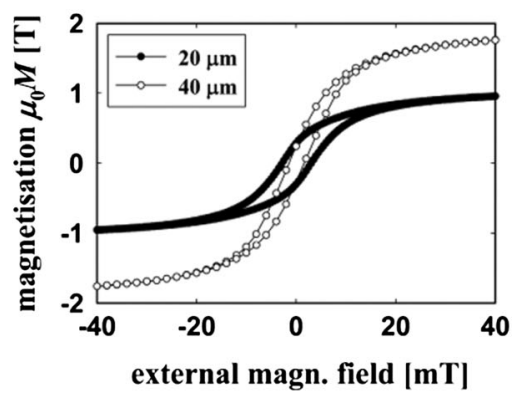

FIG. 3. (a) Phase shift of three different areas (averaged over $12 \times 12$ pixels) of the NSPI images as a function of the neutron wavelength $\lambda_{0}$ : gap (circle), $20 \mu \mathrm{m}$ foil (square), and $40 \mu \mathrm{m}$ foil (triangle). The black curves are the linear fits through the data points. (b) Hysteresis curves of the two different ferromagnetic steel foil materials measured with a PPMS.
As a second example, a $9 \mathrm{~mm}$ long cylindrical ferromagnetic rod with a diameter of $2.2 \mathrm{~mm}$ was oriented parallel to the magnetic field and imaged as described before. Figure 4(a) presents one of the 11 radiography images used to generate the NSPI image. In its center, one can clearly recognize the strongly absorbing steel rod, which causes a dipolar magnetic field in its vicinity. Dark pixels indicate a lowered neutron intensity with respect to the empty beam image, due to a neutron spin phase shift $\Delta \varphi$ of about $180^{\circ}$ modulo $360^{\circ}$. At the position of the bright pixels no net phase shift modulo $360^{\circ}$ occurs, so there the intensity is of the same size as in the empty beam image. Moreover, the lines of equal neutron intensity correspond to the magnetic field lines and show the typical dipolar shape. The NSPI image of the steel rod given in Fig. 4(b) shows three further characteristic features:

(i) The strong magnetic fields inside and very near the rod cause large phase shifts and therefore lead to a low $\mathrm{S} / \mathrm{N}$ ratio of the intensity modulation [compare Fig. 1(e)], due to the finite width of the wavelength distribution of the neutron beam. Hence, phase retrieval inside the rod is not possible and results in noise in the NSPI image.

(ii) The phase jumps (black to white jumps) in the image indicate a spin precession of more than $360^{\circ}$. Further, the decreasing distance between the individual phase jumps implies that the field gradient increases with decreasing distance to the rod, just as expected for a dipolar field.

(iii) The image shows no phase shift along the angle of $52^{\circ} \pm 2^{\circ}$, due to the angular dependent term of the dipolar field: $\left(3 \cos ^{2} \theta-1\right)$. Theoretically for a pointlike dipole this angle would be $\theta_{m}=54.7^{\circ}$ [20].

Technical details.-The NSPI technique is a combination of a neutron Ramsey apparatus and a standard radiography setup [21], consisting of a two-dimensional position sensitive detector and a well-collimated polarized and monoenergetic neutron beam. A scheme of the NSPI setup is presented in Fig. 5. The employed polarized neutron beam was provided by the small angle instrument SANS-I [22] at the spallation neutron source SINQ at the Paul Scherrer Institute. Its neutron wavelength spectrum is a triangular shaped distribution with a standard width of $\Delta \lambda / \lambda_{0}=0.10 . \lambda_{0}$ is the center of gravity of the spectrum, which can be adjusted by means of a velocity selector from 0.45 to $1.2 \mathrm{~nm}$. The beam size was restricted by a $20 \times$ $20 \mathrm{~mm}^{2}$ aperture and the beam divergence was measured to be $0.20^{\circ}$, which corresponds to a $l / d$ ratio of approxi- mately 280. The $l / d$ ratio is a figure of merit commonly used in neutron radiography, where $l$ denotes the distance between a circular pinhole diaphragm with diameter $d$ and the imaged object [23]. The sample to detector distance $L$ is limited by the length of the spin analyzer. A specially designed transmission spin analyzer optimized for neutron radiography was used [24], with which $L$ could be minimized down to $225 \mathrm{~mm}$. This yields a geometrical resolution of $0.8 \mathrm{~mm}$ (FWHM of the point spread function), which is much larger than the pixel size of $0.13 \times$ $0.13 \mathrm{~mm}^{2}$ of the detector [25]. Hence, the detector does not limit the resolution of the NSPI setup. A steady magnetic field $B_{0}$ of about $19 \mathrm{mT}$ is provided by permanent magnets and trimming coils, corresponding to a neutron Larmor frequency of about $550 \mathrm{kHz}$. The two coils of the resonance $\pi / 2$-spin-flippers are separated by about $100 \mathrm{~mm}$ from each other and produce a linear oscillating
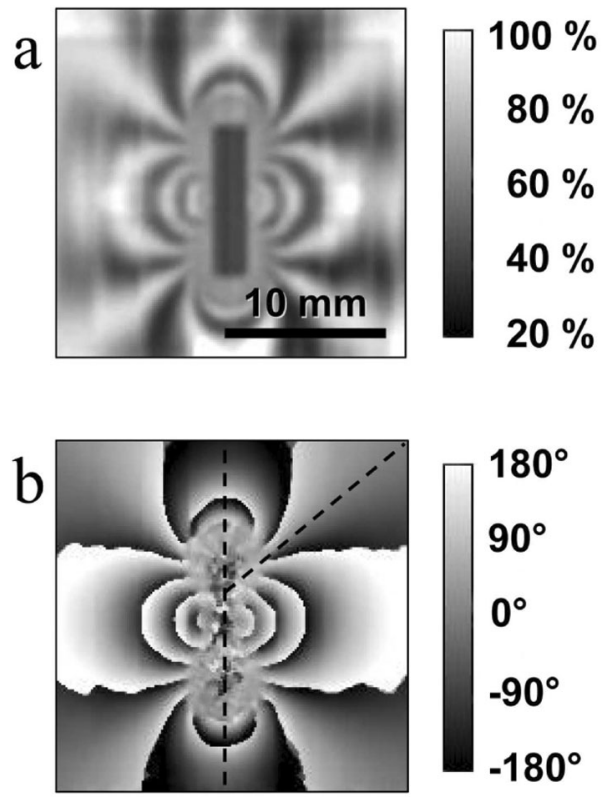

FIG. 4. (a) Radiography image of the $9 \mathrm{~mm}$ long steel rod normalized with an empty beam image, taken at a $\pi / 2$-spin flipper frequency of $550 \mathrm{kHz}$ with neutrons of $\lambda_{0}=0.5 \mathrm{~nm}$. The size of the image is $21.5 \times 21.5 \mathrm{~mm}^{2}$. (b) NSPI image generated from a set of 11 individual images. The dashed lines mark the vertical magnetic field direction and the axis along which the spin phase shift is zero due to the vanishing dipolar field under the angle of $52^{\circ} \pm 2^{\circ}$. 


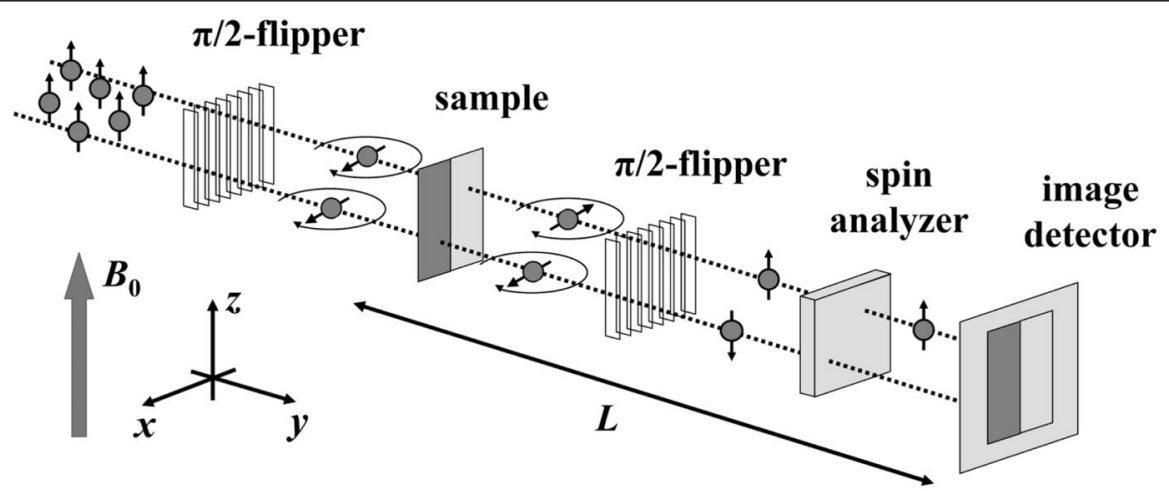

FIG. 5. Scheme of the neutron spin phase imaging setup. The spins of the incoming polarized neutron beam are primarily oriented in $z$ direction along the steady magnetic field $\vec{B}_{0}$. They pass two phase-locked $\pi / 2$-spin flipper coils, which produce a oscillating field in $x$ direction with an angular frequency $\omega$. A sample with a spin-dependent potential produces additional neutron spin precession, causing dephasing of the neutron spins. The spin analyzer lets pass only neutrons of one spin state, which get detected by the twodimensional image detector.

field in $x$ direction. The stability of the spin flippers and of the magnetic field is so far limiting the accuracy of the phase shift retrieval of the setup to approximately $\pm 1^{\circ}$ for neutrons with a wavelength of $0.5 \mathrm{~nm}$. This yields a sensitivity for the magnetic field path integral of about $\pm 7.5 \times$ $10^{-8} \mathrm{~T} \mathrm{~m}$. A more detailed description of the apparatus is given elsewhere [26].

Conclusion.-The presented examples achieved with the novel neutron spin phase imaging technique show the feasibility of quantitative imaging of magnetic fields inand outside magnetic materials with a submillimeter resolution. This is fundamentally different to another recently published approach [27], which only determines the degree of beam depolarization and therefore cannot deliver quantitative results about magnetic fields.

Furthermore, the NSPI technique also permits to apply any external magnetic field to the sample [26], which then, for example, allows to locally observe the magnetization of magnetic objects as a function of the field strength. A 2.5 Tesla NSPI setup [12] is currently used to investigate the possibility to image dia- and paramagnetic materials. The next steps will be to test other parameters, e.g., temperature, pressure and electric fields, and to perform three dimensional tomographies of magnetic fields and samples. Hence, the technique offers a wealth of new possibilities in real space condensed matter research.

*florian.piegsa@psi.ch

[1] Neutron Radiography: Proceedings of the Eighth World Conference, edited by M. Arif (Destech Pub. Inc., Lancaster, PA, 2008).

[2] E. H. Lehmann et al., Nucl. Instrum. Methods Phys. Res., Sect. A 576, 389 (2007).

[3] A. S. Tremsin et al., Nucl. Instrum. Methods Phys. Res., Sect. A 592, 374 (2008).

[4] F. M. Piegsa, Nucl. Instrum. Methods Phys. Res., Sect. A (to be published).
[5] C. Grünzweig et al., Rev. Sci. Instrum. 78, 053708 (2007).

[6] R. Golub et al., Am. J. Phys. 62, 779 (1994).

[7] M. T. Rekveldt et al., Appl. Phys. A 74, S323 (2002).

[8] N. Ramsey, Phys. Rev. 78, 695 (1950).

[9] N. Ramsey, Molecular Beams (Oxford Univ. Press, New York, 1956).

[10] F. Mezei, Z. Phys. 255, 146 (1972).

[11] H. Glättli and M. Goldman, Methods of Experimental Physics (Academic Press, New York, 1987), Vol. 23 C, p. 241.

[12] F. M. Piegsa et al., Nucl. Instrum. Methods Phys. Res., Sect. A 589, 318 (2008).

[13] A. Abragam and M. Goldman, Nuclear Magnetism: Order and Disorder (Oxford Univ. Press, Oxford, 1982).

[14] A. I. Frank et al., Phys. At. Nucl. 65, 2009 (2002).

[15] M. Hino et al., Physica (Amsterdam) 213B-214B, 842 (1995).

[16] O. Zimmer et al., Eur. Phys. J. direct A 4, 1 (2002).

[17] C. Mazure-Espejo et al., Physica (Amsterdam) 120B, 66 (1983).

[18] For further specifications, see http://www.hs-folien.de.

[19] Quantum Design: http://www.qdusa.com.

[20] See EPAPS Document No. E-PRLTAO-102-005917 for another demonstrative example presenting the radiography of the magnetic field produced by a small coil. For more information on EPAPS, see http://www.aip.org/ pubservs/epaps.html.

[21] F. M. Piegsa et al., Nucl. Instrum. Methods Phys. Res., Sect. A 586, 15 (2008).

[22] V. K. Aswal et al., Nucl. Instrum. Methods Phys. Res., Sect. A 586, 86 (2008).

[23] Neutron Radiography: Proceedings of the First World Conference, edited by J.P. Barton et al. (Reidel Pub. Comp., Dordrecht, 1983).

[24] F. M. Piegsa and M. Schneider, Nucl. Instrum. Methods Phys. Res., Sect. A 594, 74 (2008).

[25] M. J. Mühlbauer et al., Nucl. Instrum. Methods Phys. Res., Sect. A 542, 324 (2005).

[26] F. M. Piegsa et al., Nucl. Instrum. Methods Phys. Res., Sect. A (to be published).

[27] N. Kardjilov et al., Nature Phys. 4, 399 (2008). 